\title{
Balloon-borne heterodyne stratospheric limb sounder TELIS ready for flight
}

\author{
R.W.M. Hoogeveen ${ }^{1 \mathrm{a}}$, P.A. Yagoubov ${ }^{\mathrm{a}}$, G. de Lange ${ }^{\mathrm{a}}$, A. de Lange ${ }^{\mathrm{a}}$, \\ V.P. Koshelets ${ }^{\mathrm{b}}$, B.N. Ellison ${ }^{\mathrm{c}}$ and M. Birk ${ }^{\mathrm{d}}$ \\ a SRON Netherlands Institute for Space Research, Utrecht, the Netherlands \\ ${ }^{\mathrm{b}}$ Institute of Radio Engineering and Electronics, Moscow, Russia \\ ${ }^{\mathrm{c}}$ Rutherford Appleton Laboratories, Chilton, Didcot, United Kingdom \\ ${ }^{\mathrm{d}}$ Deutsches Zentrum für Luft- und Raumfahrt, DLR-IMF, Oberpfaffenhofen, Germany
}

\begin{abstract}
TELIS (TErahertz and submm LImb Sounder) is a three-channel balloon-borne heterodyne spectrometer for atmospheric research. The observational techniques of TELIS can be compared to the presently flying MLS instrument on board NASA's EOS-Aura satellite, but TELIS is built with a new generation of cryogenic heterodyne detectors and novel compact systems suitable for integration into the confined space of a balloon borne cryostat. TELIS will fly on the MIPAS-B2 gondola. The two instruments together will yield the most complete set of stratospheric constituents, measured so far. TELIS is a cooperation between the European institutes DLR (PI-institute), RAL and SRON. First flight foreseen in the spring of 2008 from Teresina, Brasil.

The three TELIS receivers provide simultaneous vertical profile measurement of a range of molecules. The $500 \mathrm{GHz}$ channel is developed by RAL and will produce vertical profiles of $\mathrm{BrO}, \mathrm{ClO}, \mathrm{O}_{3}$ and $\mathrm{N}_{2} \mathrm{O}$. The $1.8 \mathrm{THz}$ channel is developed by DLR and will mainly target the $\mathrm{OH}$ radical, and will also measure $\mathrm{HO}_{2}, \mathrm{HCl}, \mathrm{NO}, \mathrm{NO}_{2}, \mathrm{O} 3, \mathrm{H}_{2} \mathrm{O}, \mathrm{O}_{2}$ and $\mathrm{HOCl}$. Finally the $480-650 \mathrm{GHz}$ channel is developed by SRON and IREE and will measure profiles of $\mathrm{ClO}, \mathrm{BrO}, \mathrm{O}_{3}$, $\mathrm{HCl}, \mathrm{HOCl}, \mathrm{H}_{2} \mathrm{O}$ and its 3 isotopomers, $\mathrm{H}_{2} \mathrm{O}_{2}, \mathrm{NO}, \mathrm{N}_{2} \mathrm{O}, \mathrm{HNO}_{3}, \mathrm{CH}_{3} \mathrm{Cl}$ and $\mathrm{HCN}$.

In this paper, the science and technology of TELIS will be discussed with emphasis on the channel developed by SRON. It contains a Superconducting Integrated Receiver (SIR), which combines on a $4 \times 4 \mathrm{~mm}^{2}$ chip the low-noise SIS mixer and its quasioptical antenna, a superconducting phase-locked Flux Flow Oscillator (FFO) acting as Local Oscillator (LO) and a SIS harmonic mixer (HM) for FFO phase locking. The latest results from the pre-flight test and integration campaigns will be presented.
\end{abstract}

Key words: Atmospheric, limb-sounding, cryogenic, heterodyne, sub millimeter.

\section{INTRODUCTION}

The millimeter and sub-millimeter (terahertz, THz) region of the spectrum is well suited for the study of the composition of the upper Earth's upper atmosphere. Many of the atmospheric trace gases have their rotational transition lines in this spectral region. The very high resolution required (on the order of $10^{6}$ ) in order to fully exploit the spectral signature, can be obtained by heterodyne detection techniques. Heterodyne detection is well known and has been widely applied at radio frequencies, extending, in recent years, well into the terahertz frequency domian.

The principle of heterodyne detection is to add a strong monochromatic signal from a local-oscillator (LO) to the incoming atmospheric radiation. The frequency of the $\mathrm{LO}$ is chosen to be a few $\mathrm{GHz}$ away from the frequency band of interest. The combined atmospheric and LO signal is detected by a so-called mixer detector, which is a non-linear device that provides an output frequency which is the difference between the LO and the input signal originating from

\footnotetext{
${ }^{1}$ SRON, Sorbonnelaan 2, 3584 CA, Utrecht, the Netherlands. R.Hoogeveen@sron.nl, phone +31 302535703 , fax +31 302540860 ,
} www.sron.nl. 
the atmosphere. The difference frequency is referred to as the intermediate frequency (IF) and is typically in the few $\mathrm{GHz}$ range. The IF spectrum contains the same spectral information as the original sub millimeter atmospheric spectrum, but can be more easily electronically processed.

Application of the heterodyne detection technique in atmospheric observations from space has been pioneered by the Microwave Limb Sounder (MLS) on board the UARS satellite [1], operational between 1991 and 1999. UARS/MLS measured stratospheric ozone, $\mathrm{ClO}$, water vapor, pressure, and temperature using bands at 63, 183, and $205 \mathrm{GHz}$. Its improved successor MLS on board of EOS-Aura has been launched July 2004 [1] and performs very successfully. The frequency bands have been extended to $118 \mathrm{GHz}$ (for temperature and pressure), $190 \mathrm{GHz}\left(\mathrm{H}_{2} \mathrm{O}\right.$ and $\left.\mathrm{HNO}_{3}\right), 240 \mathrm{GHz}$ $\left(\mathrm{O}_{3}\right.$ and $\left.\mathrm{CO}\right), 640 \mathrm{GHz}\left(\mathrm{HCl}, \mathrm{ClO}, \mathrm{BrO}, \mathrm{HO}_{2}\right.$, and $\mathrm{N} 2 \mathrm{O}$ ), and $2.5 \mathrm{THz}(\mathrm{OH})$.

In Europe, the Swedish ODIN satellite [2] carries the Sub Millimeter Radiometer instrument which is used for atmospheric research as well as for astronomical observations. Its frequency bands are located at $118.25-119.25 \mathrm{GHz}$, 486.1 - $503.9 \mathrm{GHz}$, and 541.0 - 580.4 GHz. ODIN was launched in 2002 and is still operational.

Obviously, in any heterodyne receiver system the mixer is a key element. Traditionally, both uncooled (MLS) and cooled (100 K for ODIN/SMR) Schottky diodes have been used. Schottky mixer diodes have the advantage of cryogenfree operation, but compared to superconducting mixers (operating around $4 \mathrm{~K}$ ), they are an order of magnitude noisier and may require orders of magnitude more Local Oscillator power. At terahertz frequencies the LO-power requirement excludes the use of solid-state devices and necessitates cumbersome gas lasers: Aura/MLS carries a $\mathrm{CO}_{2}$-laser pumped methanol laser to generate the required $\mathrm{LO}$ power at $2.5 \mathrm{THz}$ [1].

Modern superconducting mixers offer huge performance improvements, especially in terms of noise and required LO power and have superseded Schottky mixers on all ground-based astronomy observatories. The two types of mixers being used are Superconductor-Insulator-Superconductor (SIS) mixers for fequencies below $1.2 \mathrm{THz}$, and Hot-Electron Bolometer mixers (HEBM) for the higher frequencies.. Two satellite instruments are currently under development that employ SIS and HEBM mixers: JEM/SMILES [3] for observing the Earth's atmosphere and Herschel/HIFI [4] for astrophysical research. The Japanese SMILES instrument (Superconducting Submillimeter-Wave Limb Emission Sounder) for the Japanese Experiment Module (JEM) of the International Space Station will be used for the observation of many atmospheric species around 625 and $650 \mathrm{GHz}$. Launch is foreseen for 2008 or beyond. The first satellite instrument to use SIS mixers will be the HIFI instrument (Heterodyne Instrument for the Far Infrared). This instrument is currently integrated onto the Herschel satellite and will be launched in 2008.

Newly proposed to the US National Research Council is the SMLS instrument (Scanning MLS) on board CAMEO [1]. Here, superconducting SIS mixers are proposed for the millimeter channel $180-280 \mathrm{GHz}$ and two sub-millimeter channels $580-680 \mathrm{GHz}$. The low-noise characteristics of these mixers reduces the integration time for a single measurement to a few milliseconds, allowing for two orders of magnitude more observations in comparison to the standard mixers used in MLS. With SMLS a full 3-D characterization of the atmosphere will be possible. When selected, CAMEO/SMLS will be launched in the 2015-2018 timeframe.

Extrapolating the current trends towards the future, we foresee Earth limb sounding from a satellite platform with superconducting receivers operating at millimeter and sub-millimeter wavelengths. Instead of gas lasers only solid-state local oscillators are to be employed to reduce system complexity. Low-noise IF-amplifiers are needed to fully exploit the sensitivity gain by the superconducting mixers. Here the trend is to employ the GaAs and InP High Electron Mobility Transistor (HEMT) amplifiers. Most likely candidates for the back-end spectrometers are Acousto-Optic Spectrometers, Digital Auto Correlators or Fast Fourier Transform Spectrometrs, in contrast to the current filter-bank spectrometers.

Several balloon and aircraft instruments anticipate space application of cryogenic technologies, as well as ground based astronomical observatories. Without being complete a few examples are given. Already operationally flying instruments for Earth observation using SIS mixers are: PIROG [5], ASUR [6] and BSMILES [7].

Three European national institutes (DLR as the PI institute, SRON and RAL) are now collaborating to develop a high sensitivity, balloon-borne atmospheric sounder that will allow simultaneous measurement of many key molecular constituents within the stratosphere. The TELIS instrument (TErahertz and sub-millimeter LImb Sounder) will provide vertical profiles of very many atmospheric trace gasses that are associated with the depletion of stratospheric ozone and climate change. The ambitious science goals of the TELIS instrument are accomplished by use of three independent frequency channels: $500 \mathrm{GHz}, 480$ - $650 \mathrm{GHz}$ and $1.8 \mathrm{THz}$. All channels will use a state-of-the-art superconducting SIS 
and Hot Electron Bolometer (HEB) mixer technology, and state-of-the-art support technology for cryogenic heterodyne detection, such as low-noise cryogenic IF amplifiers and digital auto-correlator spectrometers.

The balloon platform on which TELIS will fly also contains a Fourier transform spectrometer (MIPAS-B) developed and operated by the Institute of Meteorology and Climate research of the University of Karlsruhe, Germany [8]. MIPAS will simultaneously measure within the range 680 to $2400 \mathrm{~cm}^{-1}$. The combination of the TELIS and MIPAS instruments will provide a wealth of scientific data, both as a stand alone chemistry mission and in complement to existing spaceborne instruments, e.g., ENVISAT, Aura and METOP.

At present, the instrument is fully assembled and tested. The first flight of the TELIS instrument is foreseen in the spring of 2008.

In the next sections, first the science of TELIS will be discussed. Next the TELIS instrument description will be given in section 3 and details of the SIR channel in section 4. The latest experimental results are discussed in section 5. Conclusions will be given in the last section.

\section{THE SCIENCE OF TELIS}

An extensive list of molecular species will be targetted by TELIS: $\mathrm{BrO}, \mathrm{ClO}, \mathrm{HCl}, \mathrm{HOCl}, \mathrm{CH}_{3} \mathrm{Cl}_{1} \mathrm{O}_{3}$ (normal and isotopic), $\mathrm{H}_{2} \mathrm{O}$ (normal and isotopic), $\mathrm{OH}, \mathrm{HO}_{2}, \mathrm{HNO}_{3}, \mathrm{NO}, \mathrm{N}_{2} \mathrm{O}, \mathrm{NO}_{2}, \mathrm{HCN}$, and $\mathrm{O}_{2}$. Vertical profiles of even very weak individual lines can be determined as the TELIS receivers combine limb sounding with a high frequency resolution and low noise observations.

With respect to stratospheric ozone depletion both the halogen chemistry and $\mathrm{HO}_{\mathrm{x}}$ chemistry can thoroughly be investigated. Ozone isotopes may give insight in hidden and unknown chemical reactions and isotopic water may shed light on the origin of stratospheric water, which is connected to ozone through the $\mathrm{HO}_{\mathrm{x}}$ cycle and Polar Stratospheric Clouds (PSCs).

A key question in stratospheric sciences is whether ozone will recover in the coming decades as a result of international regulations on ozone depleting substances. In the lower and middle stratosphere ozone depletion is governed by halogen chemistry (chlorine and bromine), which is fairly well understood. The fact that TELIS and MIPAS can retrieve almost all species appearing in the catalytic halogen ozone depletion cycles will put the existing atmospheric chemistry models to stringent tests. In the upper stratosphere also $\mathrm{HO}_{\mathrm{x}}$ and $\mathrm{NO}_{\mathrm{x}}$ become important catalytic ozone depletion forces. Here atmospheric chemistry models are less accurate: ozone concentrations are underpredicted, $\mathrm{OH}$ is underpredicted, and $\mathrm{HO}_{2}$ is overpredicted. Observing all species simultaneously will shed light upon the production and loss mechanisms of $\mathrm{HO}_{\mathrm{x}}$ and the partitioning between $\mathrm{OH}$ and $\mathrm{HO}_{2}[9,10]$.

Stratospheric water vapor plays an important role in the ozone chemistry as a source gas for the production of $\mathrm{HO}_{\mathrm{x}}$. The origin is of stratospheric water is still not completely understood. Stratospheric water is formed in situ by the oxidation of species, for instance, methane, and is transported from the troposphere through the limiting cold trap of the tropopause. The accurate measurement of water isotopologues may give insight in the relative weights of the water loading mechanisms of the stratospheric, as the different masses and energy level structures of the isotopologues result in differences in evaporation, condensation, and chemical reactions.

\section{TELIS INSTRUMENT DESCRIPTION}

A complete design drawing of the TELIS instrument is shown in Figure 1. The optical front-end of TELIS is common for the three channels and consists of a pointing telescope, calibration blackbody, and relay and band-separating optics. Details of the optical design can be found in $[11,12,13]$. The telescope is a dual offset Cassegrain antenna. All three telescope mirrors are mounted on a common frame that can be rotated around the axis coinciding with the direction of the output beam. The vertical (elevation) resolution at the tangent point is about $2 \mathrm{~km}$ at $500 \mathrm{GHz}$ (FWHM), inversely proportional to the frequency. The limb scans range from upper troposphere $(10 \mathrm{~km})$ to the flight altitude at about $37 \mathrm{~km}$ with 1 to $2 \mathrm{~km}$ steps. Horizontal (azimuth) resolution is about a factor of 2 worse due to the anamorphicity of the telescope but not of prime importance for this mission as the atmospheric properties within the beam hardly depend on the azimuth. 


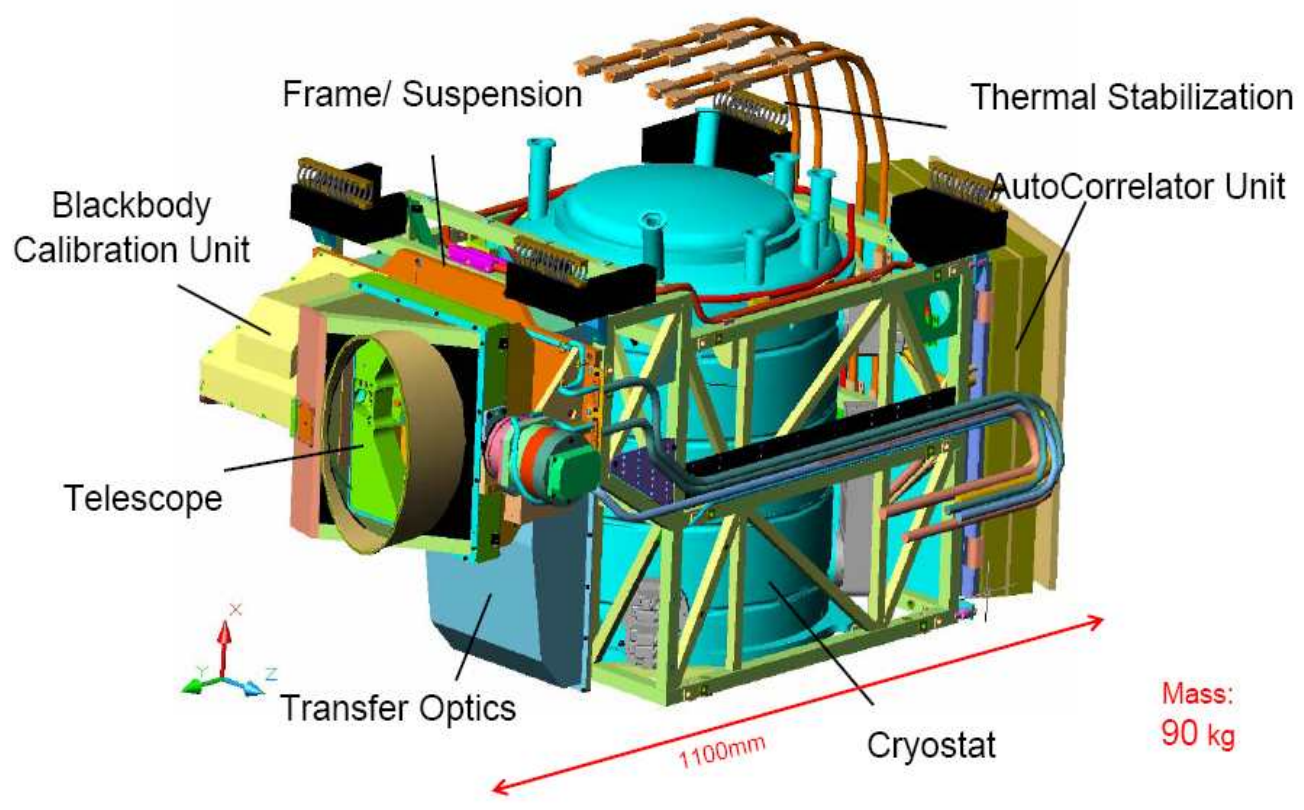

Figure 1: Design drawing of the complete TELIS instrument.

Calibration of the radiometric gain of the spectrometers is done with two blackbody reference sources. The hot-load consists of a conical black body at the ambient temperature that can be viewed by a small switching mirror in the warm optics. The cold sky reference is measured with the telescope set at 60 degree upwards with respect to the limb position. The two references are measured once or twice in every antenna scan.

Frequency separation between the channels is performed quasi-optically, allowing simultaneous observations by all receivers. For this, the beam is first split in two polarization components by a wire grid. The $500 \mathrm{GHz}$ channel uses the reflected beam. The beam is then split by a dichroic filter. After the splitting, the three beams enter a custom designed liquid-helium cooled cryostat. A number of off-set reflectors are used to interface the optics from the telescope to the cryogenic channels.

Inside the cryostat, each receiver has dedicated cold optics, mixing element and IF amplifiers.

The $500 \mathrm{GHz}$ receiver channel is being developed by RAL [14], see Figure 2. It is a highly compact unit consisting of a fixed-tuned waveguide SIS mixer, cryogenic solid-state local oscillator (LO) chain and a low-noise IntermediateFrequency (IF) chain. Single sideband ${ }^{2}$ operation is achieved through the use of a miniature cryogenic dichroic filter that provides a $4 \mathrm{~K}$ image termination and image band rejection of $>25 \mathrm{~dB}$. For optimization of the performance of the dichroic single-sideband filter a high IF is chosen: $14-18 \mathrm{GHz}$.

The 480-650 GHz receiver channel is being developed in cooperation between IREE and SRON and is based on a single-chip Superconducting Integrated Receiver (SIR) that comprises on one substrate a low-noise SIS mixer with quasi-optical antenna and a superconducting Flux Flow Oscillator (FFO) acting as LO [15, 16, 17]. Tunability of the FFO shall allow for a wideband operation of this channel, with a goal to obtain $170 \mathrm{GHz}$ instantaneous rf bandwidth or even more. The SIR channel is discussed in detail in the next section.

The $1.8 \mathrm{THz}$ channel is based on a phonon-cooled NbN HEB Mixer technology and is being developed by DLR, also acting as Principle Investigator (PI) for the TELIS mission. It is similar to that under development for SOFIA by MSPU and DLR [18]. It will utilize a cryogenic solid-state LO that is loss-less coupled to the mixer via an optical interferometer (Martin Puplett type).

\footnotetext{
${ }^{2}$ In heterodyne detection the difference signal between the Local Oscillator and the atmospheric signal is amplified. As this difference can be positive and negative, two frequency sidebands above and below the LO are detected simultaneously. Singlesideband filters transmit the signal band of the atmospheric signal and block the image sideband.
} 


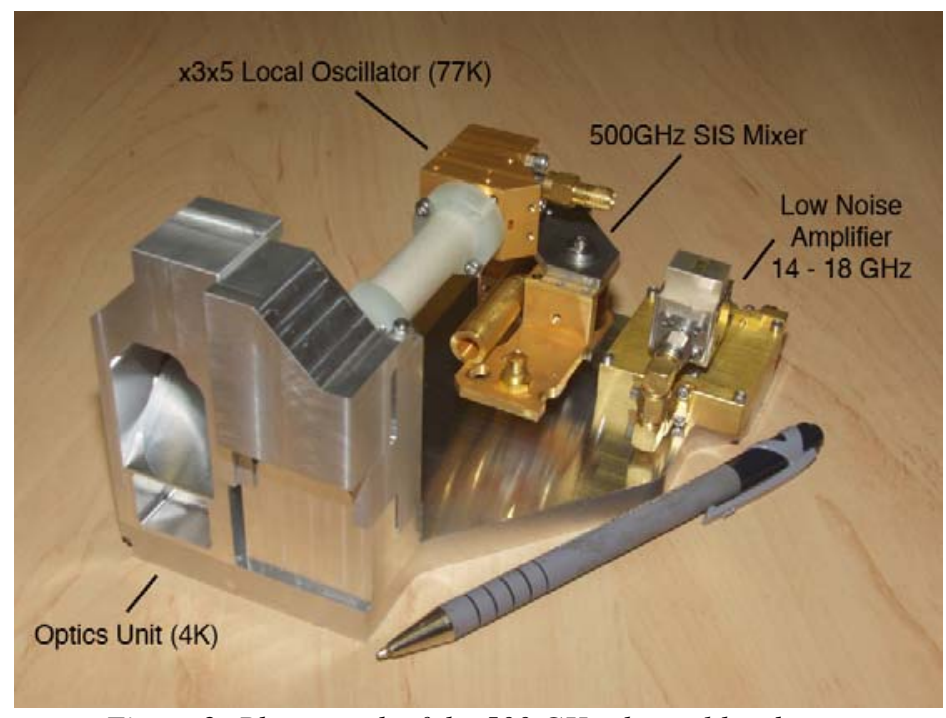

Figure 2: Photograph of the $500 \mathrm{GHz}$ channel hardware.

Three amplified output IF signals are fed to an IF processor which converts the IF to the input frequency range of the digital autocorrelator of two times $2 \mathrm{GHz}$ bandwidth. Both IF processor and digital autocorrelator are developed by the Swedish Omnisys company [19].

An on-board PC-104 computer interfaces with the control electronics of the three receiver channels and the instrument, with the digital auto correlator, with the host instrument MIPAS, and with the ground segment through a radio link. The ground segment consists of a server computer interfacing with three dedicated client computers through TCP/IP socket connections.

The complete system is battery powered and is designed for a 24 hour flight.

\section{THE SUPERCONDUCTING INTEGRATED RECEIVER}

The most challenging part of the SIR channel is by far the phase-locked Superconducting Integrated Receiver (SIR) chip developed at IREE [15, 16, 17]. SIR comprises on one $4 \times 4 \times 0.5 \mathrm{~mm}^{3}$ chip a low-noise SIS mixer with quasioptical antenna, a Flux Flow Oscillator (FFO) acting as Local Oscillator (LO) and a SIS Harmonic Mixer (HM) for FFO phase locking, see Figure 3.

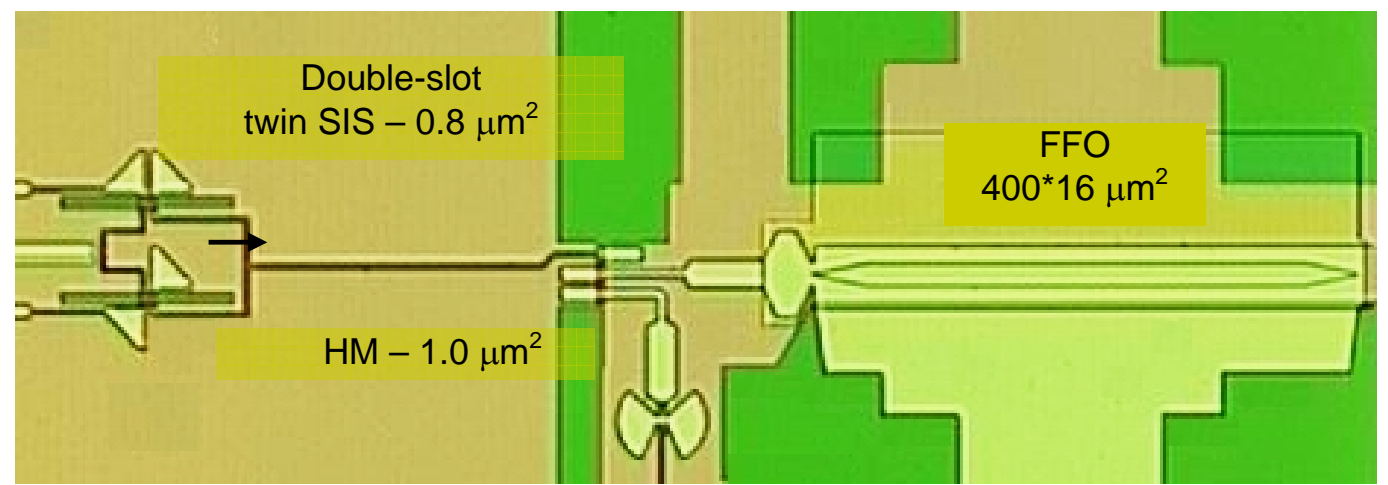

Fig. 3. Microphotograph of the central part of the SIR chip with double slot antenna. 
All components of the SIR microcircuits are fabricated in a high quality Nb-AlN/NbN-Nb tri-layer on a Si substrate [20]. The receiver chip is placed on the flat back surface of the Silicon lens, forming an integrated lens-antenna. As the FFO is very sensitive for the external electromagnetic interferences the SIR chip has to be placed inside two cylindrical shields. The outer shield is made from cryo-perm and the inner shield is copper covered with $100 \mu \mathrm{m}$ of superconducting lead.

The spectral resolution of the TELIS backend spectrometer is $3 \mathrm{MHz}$, in order to resolve the exact shape of the atmospheric lines. The FFO lineshape and stability should ideally be much better than this. As the free-running linewidth of the FFO can up to $10 \mathrm{MHz}$, the FFO is locked to an external reference oscillator using a Phase Lock Loop (PLL) system. For this, a small fraction of the FFO power is directed to a so-called Harmonic Mixer (HM). The scheme of this system is shown in Figure 4. The HM is pumped by a tunable reference frequency in the range of $19-21 \mathrm{GHz}$ from the Local oscillator Source Unit (LSU), phase locked to the internal ultra stable $10 \mathrm{MHz}$ Master Oscillator. The HM mixes the FFO signal with the n-th harmonic of the $19-21 \mathrm{GHz}$ reference. The frequency of the LSU is chosen such that the difference frequency signal is about $4 \mathrm{GHz}$. This signal is amplified by a cryogenic low-noise HEMT amplifier, and downconverted to $400 \mathrm{MHz}$, where its frequency and phase are compared with a reference of $400 \mathrm{MHz}$. Both reference signals at 3.6 GHz and at $400 \mathrm{MHz}$ are phase locked to the $10 \mathrm{MHz}$ Master Oscillator.

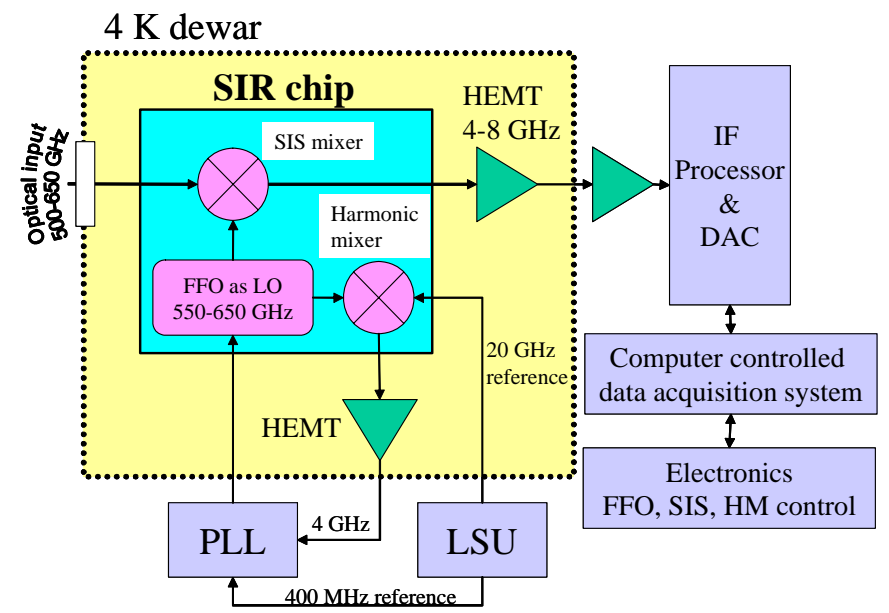

Fig. 4. Schematics of the SIR with a phase-locked LO. The FFO frequency is mixed in the Harmonic Mixer with the 19$21 \mathrm{GHz}$ reference. The mixing product is amplified, down converted and compared with the $400 \mathrm{MHz}$ reference in the $P L L$. The phase difference signal generated by PLL is used to feedback the FFO control line.

Finally, the phase difference signal generated by the PLL is used as a feedback to the FFO control-line current to compensate for the phase error. Wideband operation of the PLL (15 MHz full width) is obtained by minimizing the cable loop length. The result of the PLL on the FFO spectrum measured at one of the flight frequencies selected for TELIS is shown in Figure 5. The impact of the non-perfect FFO spectrum on the retrieval accuracy is discussed in [21].

TELIS is setup to measure spectra in the sub-millimeter and THz range. From the spectra vertical profiles of trace gases will be determined in an off-line retrieval process. The retrieval is based on the comparison between a calculated spectrum and the measured spectrum [21]. The emission spectrum is calculated in a so-called forward model by integrating the radiative transfer equation along the line of sight (LoS), for a given temperature and pressure profile, for assumed molecular density height profiles, and for the observation geometry. The instrument model convolves the spectra with the Field of View (FoV) and the instrumental line shape to model the TELIS spectra. Then, the calculated spectrum will be compared to the actual measured spectrum and the molecular density profiles will be altered to iteratively obtain the best match between modeled and observed spectrum. All spectra of a full limb scan are evaluated simultaneously to enhance the accuracy for the height profile. 


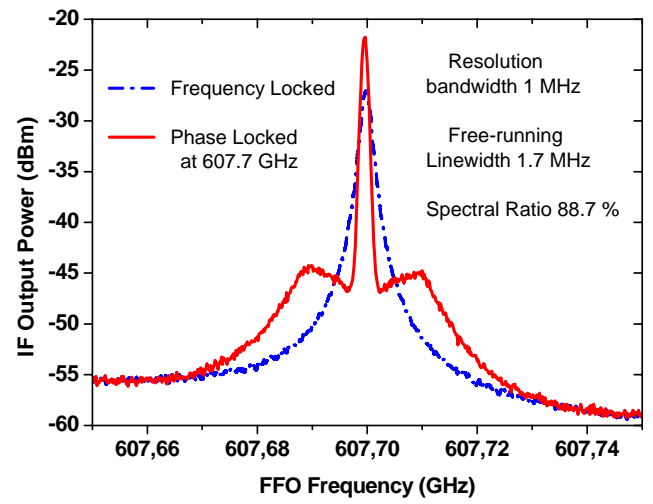

Figure 5: Spectrum of a frequency-locked FFO (blue curve) and phase-locked FFO (red). Due to limitations of the spectrum analyser the central delta-peak of the PL-FFO appears broadened.

\section{LATEST EXPERIMENTAL RESULTS}

The TELIS-SIR channel has been integrated and tested in the TELIS flight model. All experimental results discussed here have been obtained with the SIR device selected for the first flight. The measured Double-Side Band (DSB) uncorrected receiver noise temperature is given in figure $6 \mathrm{a}$ as a function of frequency, and in Figure $6 \mathrm{~b}$ as a function of IF frequency.

The red curve of Figure 6a is taken of the mixer in a dedicated dewar with only a vacuum window. The blue curve is taken with the mixer installed in the receiver, including 6 mirror reflections and including 15\% optical spill over. As can be seen, the noise is around $400 \mathrm{~K}$ over a $130 \mathrm{GHz}$ input bandwidth of the receiver, with a minimum of $300 \mathrm{~K}$ at 600 GHz. The noise peak around $540-575 \mathrm{GHz}$ is partially caused by absorption of water vapor in the path between calibration sources and cryostat, and partly due to antenna properties of the mixer. The relatively high noise in this band is not a concern as this part of the atmospheric spectrum is not usable due to an enormously strong and broadened watervapor line at $557 \mathrm{GHz}$.

After these measurements have been taken, system improvements have been implemented (a better AR coated vacuum window and better thermal filters), leading to a reduction of the minimum receiver DSB noise temperature to $200 \mathrm{~K}$. The noise as a function of IF is fairly flat in the designed frequency range 5-7 GHz, as can be seen in Figure 6b, taken after the system improvements.
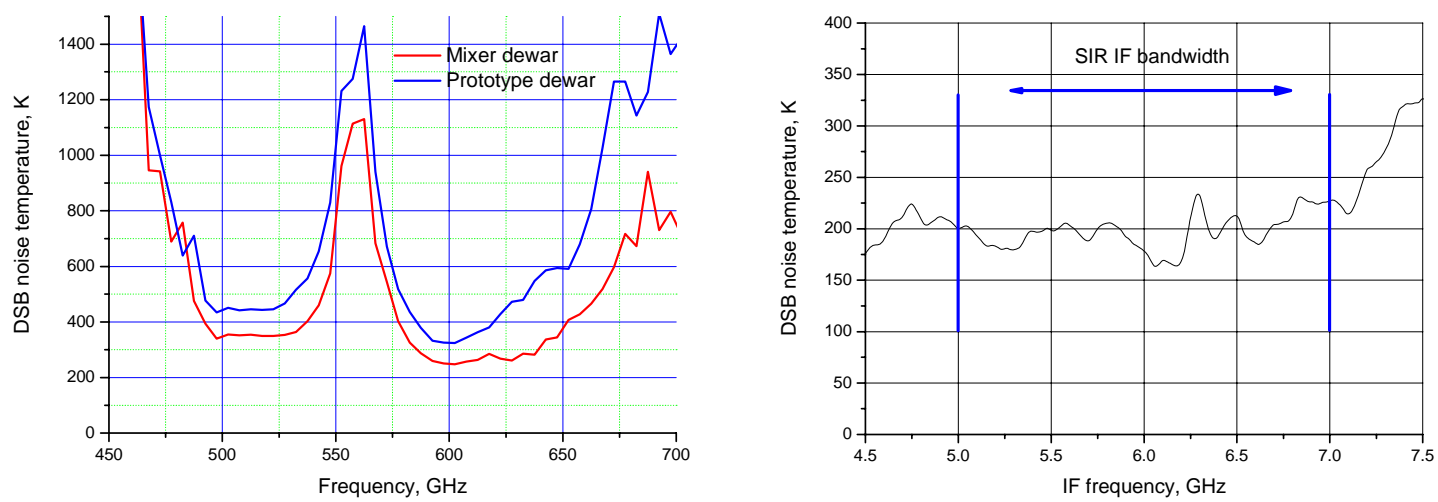

Figure 6: Left the meausred noise of the SIR device selected for flight. The noise curve in red is only for the mixer, while in blue the mixer is mounted in its receiver unit consisting of six extra mirrors. At the right the mixer noise as a

function of IF frequency, taken at $600 \mathrm{GHz}$. 
The near field beam pattern of the SIR cold channel has been measured at $600 \mathrm{GHz}$. Results of the amplitude-phase distribution are shown in Fig. 7. The measured beam waist is $2.25 \mathrm{~mm}$ (within $1 \%$ of the designed value), Gaussisity of the measured beam is $92.4 \%$.
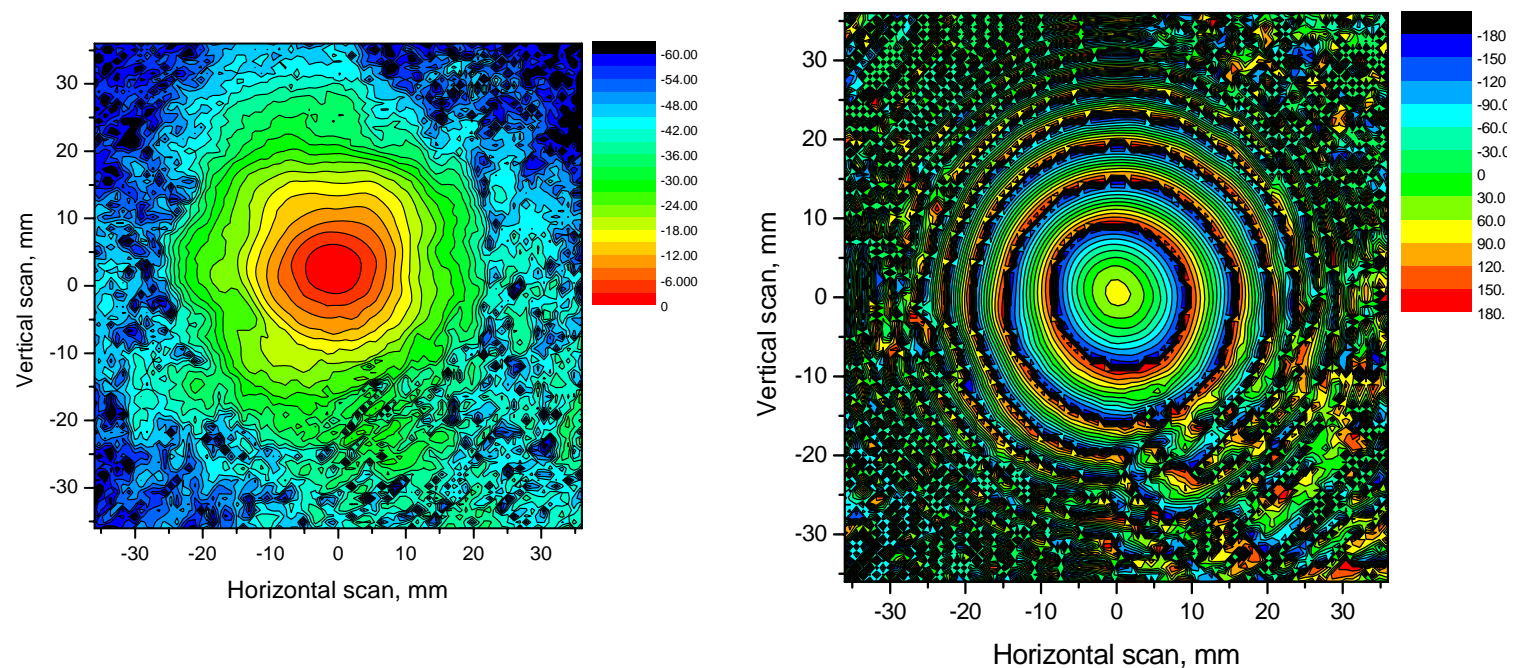

Fig. 7. SIR cold channel amplitude (left pannel) and phase (right panel) distribution. Distance from the beam waist is $110 \mathrm{~mm}$. Frequency is $600 \mathrm{GHz}$.

For the measurement strategy it is important to know the stability of the complete receiver chain. The stability determines the measurement time and thus the frequency of the calibration cycle. The stability of the complete TELISSIR system has been determined with a noise-fluctuation bandwidth of $17 \mathrm{MHz}$ and the results are presented in Fig. 8. For the two IF channels that are used to determine the Allan variance it is found that the Allan stability time is about 13.5 seconds. When the difference of the two channels is taken to determine the Allan variance (this is the so-called spectroscopic (differential) mode), an Allan-stability time of 20 seconds is found.

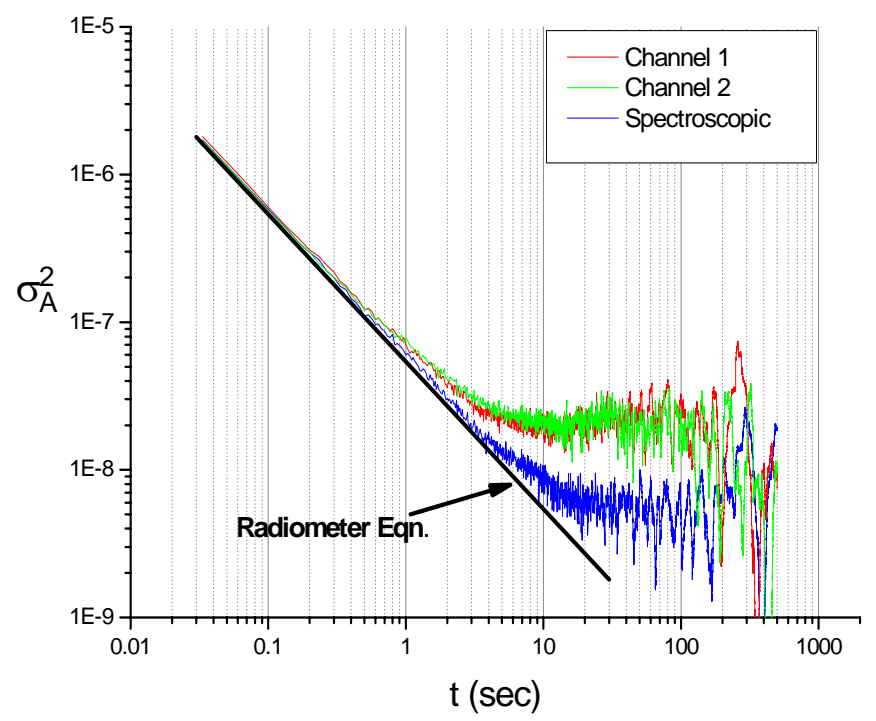

Fig. 8. System stability of the SIR channel. FFO is phase locked at $600 \mathrm{GHz}$. Green and blue lines present individual channels variance, the black one is representative for the spectroscopic variance. 
To prove the capabilities of the TELIS-SIR channel for high resolution spectroscopy we have successfully measured line profiles of OCS gas around $625 \mathrm{GHz}$. The tests were done in a laboratory gas cell setup at a gas pressure down to $0.2 \mathrm{mBar}$, corresponding to the FWHM linewidth $<5 \mathrm{MHz}$. The FFO frequency was tuned to $624.93 \mathrm{GHz}$ so that the two OCS lines at $619.621 \mathrm{GHz}$ and $631.743 \mathrm{GHz}$ have their lines in the $5-7 \mathrm{GHz}$ IF range, one in the upper side band, one in the lower side band. The measured (and deconvolved) spectrum at a gas pressure of $0.2 \mathrm{mBar}$ is shown in Fig. 9. The flat lower level of the spectrum is due to the $77 \mathrm{~K}$ cold reference and the residual emission of the warm windows of the gas cell. Both lines are calculated to be saturated. Therefore, the signal level is expected at $210 \mathrm{~K}$ for an ideal receiver. The deviation from this value is due to the sideband ratio of the receiver deviating from 1.0.

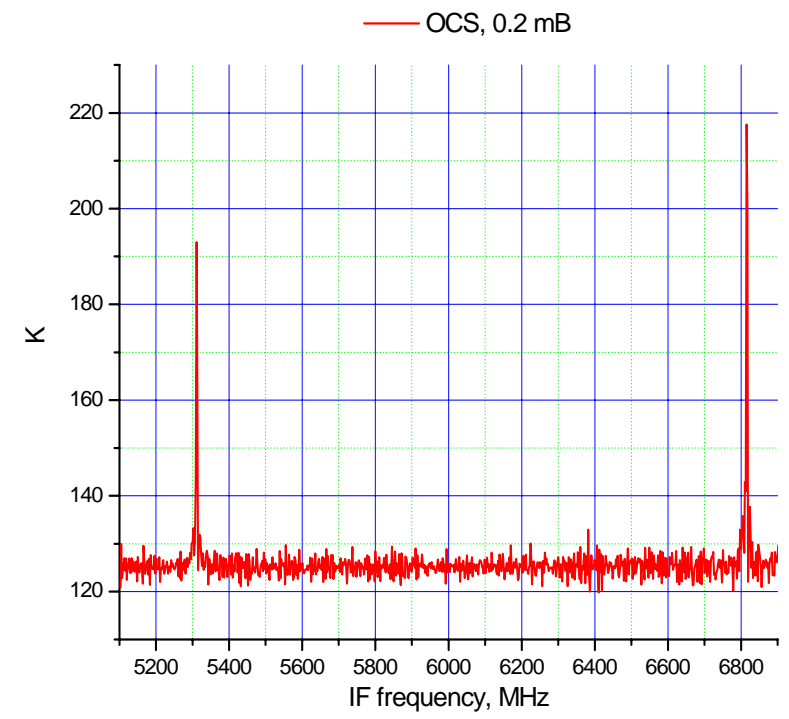

Fig. 9. Deconvolved spectrum of the OCS emission lines at a gas pressure 0.2 mBar. LO frequency $624.93 \mathrm{GHz}$. Two lines are detected, one in the lower side band, the other one in the upper side band.

\section{CONCLUSIONS}

The present status of the TELIS instrument is that we are nearly ready for flight. On instrument level some debugging, optimization and characterization needs to be done. The TELIS-SIR channel is fully ready. The first combined MIPASTELIS flight is foreseen for Spring 2008, from Teresina, Brasil.

\section{ACKNOWLEDGMENT}

The authors thank colleagues at SRON and DLR for help and assistance in the SIR channel characterization: A. Baryshev, J. Barkhof (amplitude-phase beam measurements), J. Kooi, M. Romanini (stability test), G. Wagner (gas cell measurements). T. de Graauw, A. Selig and W. Wild are acknowledged for their support in this work.

\section{REFERENCES}

1. For info on UARS/MLS, EOS-Aura /MLS and CAMEO/SMLS see: http://mls.jpl.nasa.gov/

2. U. Frisk et al., The Odin satellite: Radiometer design and test, Astronomy\&Astrophysics A\&A 402, L27-L34 (2003), and http://www.ssc.se/?id=7180

3. For info on JEM/SMILES see: http://smiles.tksc.jaxa.jp/

4. For info on Herschell/HIFI see: http://www.sron.nl/divisions/lea/hifi/ 
5. J. R. Pardo, L. Pagani, G. Olofsson, P. Febvre, and J. Tauber, "Balloonborne submillimeter observations of upper stratospheric $\mathrm{O}_{2}$ and $\mathrm{O}_{3}$ ”, J. Quant. Spectrosc. Radiat. Transf., vol. 67, pp. 169-180, 2000 and Deschamps A et al. "A balloon experiment searching for the $425 \mathrm{GHz} O 2$ line with an SIS Receiver”, proceeding of the ESA Workshop on Millimeter Wave Technology and Applications, Espoo, Finland, May 1998.

6. For info on ASUR see: http://www.iup.physik.uni-bremen.de/asur/asurhome_e.html

7. For info on SMILES see: http://www2.nict.go.jp/dk/c214/bsmiles/

8. For info on MIPAS-B see http://www-imk.fzk.de/asf/mipas-b/mipas-b.htm

9. R.R Conway, M.E. Summers, M.H. Stevens, J.G. Cardon, P. Preusse, and D. Offermann, "Satellite observations of upper stratospheric and mesospheric OH: The HOx dilemma", Geophysical Research Letters 27 (17), (2000), pp. 2613-2616.

10. T. Canty, H.M. Pickett, R.J. Salawitch, K.W. Jucks, W.A. Traub, J.W. Waters, "Stratospheric and mesospheric HOx: results from aura MLS and FIRS-2", Geophyscial Research Letters 33 (12), Art. No. L12802, 2006.

11. P. Yagoubov, H. van de Stadt, R. Hoogeveen, V. Koshelets, M. Birk, and A. Murk, "Optical design of submillimeter spectrometer for limb sounder”, in 28th ESA Antenna Workshop on Space Antenna Systems and Technologies, Noordwijk (NL), May 2005.

12. P.A. Yagoubov, W.-J. Vreeling, H. van de Stadt, R.W.M. Hoogeveen, O.V. Koryukin, V. P. Koshelets, O.M. Pylypenko, A. Murk, “550-650 GHz spectrometer development for TELIS”, proceedings of the $16^{\text {th }}$ Intern. Conf. on Space Terahertz Technology, Göteborg, Sweden, May 2-4, 2005.

13. A. Murk, P. Yagoubov, U. Mair, M. Birk, G. Wagner, H. van de Stadt, R. Hoogeveen, and N. Kämpfer, "Antenna simulations for the THz and submm limb sounder TELIS", in 28th ESA Antenna Workshop on Space Antenna Systems and Technologies, Noordwijk (NL), May 2005.

14. B.N. Ellison, B.P. Moyna, D.N. Matheson, A. Jones, S.M.X. Claude, C. Mann, B.J. Kerridge, R. Siddans, R. Munro, and W.J. Reburn, "Development of a high sensitivity airborne SIS receiver to detect ClO and BrO”, Proceedings of 2nd ESA Workshop on Millimetre Wave Technology and Applications, Espoo, Finland, May 1998.

15. V.P. Koshelets, P.N. Dmitriev, A.B. Ermakov, L.V. Filippenko, O.V. Koryukin, A.V. Khudchenko, M.Yu. Torgashin, P.A. Yagoubov, R.W.M Hoogeveen, and W. Wild, "Superconducting Submm Integrated Receiver with Phase-Locked Flux-Flow Oscillator for TELIS”, proceedings of the $16^{\text {th }}$ Intern. Conf. on Space Terahertz Technology, Göteborg, Sweden, May 2-4, 2005, and http://www.cplire.ru/html/lab234 /publications.htm

16. V.P. Koshelets, P.N. Dmitriev, A.B. Ermakov, A.S. Sobolev, M.Yu. Torgashin, V.V. Kurin, A.L. Pankratov, J. Mygind,, “Optimization of the Phase-Locked Flux-Flow Oscillator for the Submm Integrated Receiver”, “IEEE Trans. on Appl. Supercond.”, vol. 15, pp. 964-967, 2005.

17. V.P. Koshelets, A.B. Ermakov, L.V. Filippenko, A.V. Khudchenko, O.S. Kiselev, A.S. Sobolev, M.Yu. Torgashin, P.A. Yagoubov, R.W.M. Hoogeveen, and W. Wild, "Iintegrated Submillimeter Receiver for TELIS”, IEEE Trans. on Appl. Supercond., vol. 17, pp. 336-342, 2007.

18. H. Richter, A. Semenov, H.-W. Hübers, K.V. Smirnov, G.N. Goltsman, B.M. Voronov, "Phonon Cooled HotElectron Bolometric Mixer for 1-5 THz”, 2004 Joint $29^{\text {th }}$ Int. Conf. on Infrared and Millimeter Waves and $12^{\text {th }}$ Int. Conf. on Terahertz Electronics, pages 241-244.

19. See http://www.omnisys.se/

20. M.Yu. Torgashin, V.P. Koshelets, P.N. Dmitriev, A.B. Ermakov, L.V. Filippenko, and P.A. Yagoubov, "Superconducting Integrated Receivers based on Nb-AlN-NbN circuits“, IEEE Trans. on Appl. Supercond., vol. 17, pp.379- 382, 2007.

21. R. W. M.Hoogeveen; P. A. Yagoubov; A. de Lange; A. M. Selig; V. P. Koshelets; B. N. Ellison; M. Birk, “Superconducting integrated receiver development for TELIS", SPIE Proceedings Vol. 5978 (2005) 59781F 